\title{
Clinical and Laboratory Aspects of Thyroglobulin and Thyroglobulin Antibody in Differentiated Thyroid Carcinoma
}

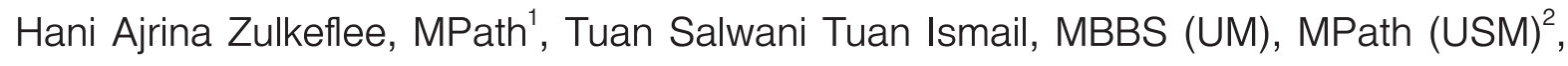 \\ Noorazliyana Shafii, MPath ${ }^{3}$
}

${ }^{1}$ Faculty of Medicine and Health Sciences, Universiti Sains Islam Malaysia, Bandar Baru Nilai, 71800 Nilai, Negeri Sembilan, Malaysia.

${ }^{2}$ Department of Chemical Pathology, School of Medical Sciences, Universiti Sains Malaysia, 16150, Kubang Kerian, Kelantan, Malaysia.

${ }^{3}$ Endocrine laboratory, Hospital Universiti Sains Malaysia, 16150 Kubang Kerian, Kelantan, Malaysia.

Received 7 September 2021 • Revised 3 December 2021 • Accepted 7 December 2021 • Published online 9 February 2022

\begin{abstract}
:
Thyroglobulin (Tg) is a precursor for thyroid hormone, specifically synthesized by thyroid follicular cells upon stimulation by the thyroid stimulating hormone (TSH). Damage to the thyroid cells caused by benign or malignant thyroid diseases cause the release of $\mathrm{Tg}$ into circulation. Differentiated thyroid cancer (DTC) is the most common endocrine malignancy, which has an excellent prognosis if detected and treated early. This review describes the clinical and laboratory aspects of $\mathrm{Tg}$ and AntiTg measurement in the management of DTC. The serum Tg measurement has been used in the pre- and post-operative management of DTC. The clinical significance of $\mathrm{Tg}$ and the role its low level can play in the diagnosis and monitoring of DTC as well as in the prediction of its recurrence must be understood. DTC may lead to the production of a different $\mathrm{Tg}$ with novel immunogenic cancer epitopes that can induce $\mathrm{TgAb}$ synthesis. The preoperative TgAb level has shown a high predictive value for DTC, while postoperative serum TgAb can be used for the detection of disease persistence and recurrence. Despite the importance of TgAb level in DTC management, the presence of TgAb interferes with the analysis of $\mathrm{Tg}$ measurement, thus limiting its clinical utility. There is no established value regarding at which level TgAb interferes with Tg measurement. Available methods in measuring Tg and TgAb should emphasize the lower and upper limits of their detection, especially for postoperative monitoring and early disease recurrence detection.
\end{abstract}

Contact: Tuan Salwani Tuan Ismail, MBBS (UM), MPath (USM)

Department of Chemical Pathology, School of Medical Sciences,

Universiti Sains Malaysia, 16150, Kubang Kerian, Kelantan, Malaysia.

E-mail: tusti@usm.my

This is an open access article under the CC BY-NC-ND license

(http://www.jhsmr.org/index.php/jhsmr/about/editorialPolicies\#openAccessPolicy).

J Health Sci Med Res 2022;40(5):589-597 doi: $10.31584 /$ jhsmr.2022862 www.jhsmr.org 
The accurate measurement of serum $\mathrm{Tg}$ and $\mathrm{TgAb}$ is pertinent to the follow-up of DTC patients, and any suspicious results must be interpreted in accordance with clinical findings in view of a present possible assay interference.

Keywords: antithyroglobulin, differentiated thyroid cancer, thyroglobulin

\section{Introduction}

Differentiated thyroid cancer (DTC) is a malignant proliferation of the follicular cells of the thyroid gland, and it includes papillary thyroid carcinoma, follicular thyroid carcinoma, and Hurthle cell carcinoma. Although it is the most prevalent endocrine malignancy ${ }^{1}$, it has an excellent prognosis if it is detected and treated early. The current management of DTC has incorporated the measurement of serum thyroglobulin $(\mathrm{Tg})$ as a primary thyroid tumor marker for treatment monitoring. ${ }^{2,3}$

$\mathrm{Tg}$ is a glycoprotein that is synthesized specifically by thyroid follicular cells as well as DTC cells. ${ }^{4}$ It has a heterogenous structure due to its post-translational modifications as well as the unregulated synthesis of mature thyroglobulin in thyroid tumor cells. ${ }^{5,6}$ Physiologically, Tg is important, as it serves as a precursor for thyroid hormone synthesis. Upon stimulation by the thyroid stimulating hormone (TSH), its synthesis is upregulated and followed by its iodination, the coupling of iodinated tyrosine residues, and the thyroid hormone secretion. ${ }^{7}$ Damage to the thyroid gland, i.e., benign or malignant thyroid diseases, can release Tg into circulation. ${ }^{8}$

Anti-thyroglobulin antibody ( $\operatorname{TgAb})$ is an immunoglobulin formed against endogenous $\mathrm{Tg}$ upon injury to the thyroid gland. ${ }^{9,10} \mathrm{TgAb}$ is heterogenous due to the varying structures of endogenous $\mathrm{Tg}$ with multiple immunogenic epitopes. ${ }^{6}$ They are detected roughly in about $10.0 \%$ of the normal population and in $30.0 \%$ of patients with DTC. ${ }^{11}$

\section{Discussion}

\section{Clinical aspects of thyroglobulin}

The preoperative $\mathrm{Tg}$ concentration offers a limited diagnostic advantage in patients presenting with thyroid nodules because the concentration of $\mathrm{Tg}$ with an intact thyroid gland is confounded by: 1) the mass of thyroid tissue; 2) thyroid gland injury, e.g., due to fine needle aspiration cytology or inflammation; and 3 ) the degree of $\mathrm{TSH}$ receptor stimulation. ${ }^{5}$ This is in line with the 2015 American Thyroid Association Management Guidelines for Adult Patients with Thyroid Nodules and Differentiated Thyroid Cancer, which does not recommend routine serum thyroglobulin measurement. ${ }^{2}$ However, a few studies have suggested that it is useful as a diagnostic marker at a certain cutoff value. A prospective study in India among 92 patients presenting with thyroid swelling revealed that a $\mathrm{Tg}$ level value of more than $53 \mathrm{ng} / \mathrm{ml}$ predicted malignancy risk with a sensitivity of $72.0 \%$ and a specificity of $73.0 \%{ }^{12}$ Another study in Turkey found that a serum $\mathrm{Tg}$ level of more than $188.5 \mathrm{ng} / \mathrm{ml}$ was associated with a higher prevalence of DTC. ${ }^{13}$ The Tg cutoff of $100 \mathrm{ng} / \mathrm{ml}$ had a sensitivity of $48.7 \%$ and a specificity of $90.0 \%$ in diagnosing follicular thyroid carcinoma among 125 Chinese patients with thyroid tumor. ${ }^{14}$ Nevertheless, the suggested $\mathrm{Tg}$ cutoff levels have varied widely between populations; therefore, further studies are required to establish and validate a standardized limit that can be utilized in medical decision-making. 
Traditionally, the initial assessment of tumor staging in patients diagnosed with DTC employs/involves a radiological modality such as ultrasonography. ${ }^{15}$ However, it is highly operator-dependent and susceptible to the under evaluation of surrounding lymph nodes. This may lead to a suboptimal treatment plan and can predispose patients to a higher risk of disease recurrence. Several studies have demonstrated the utility of pre-operative serum $\mathrm{Tg}$ level in assessing both tumor load and distribution in confirmed DTC cases. A study among 57 Korean patients with DTC found that a preoperative serum $\mathrm{Tg}$ level greater than 63.4 $\mathrm{ng} / \mathrm{ml}$ had a sensitivity of $84.2 \%$ and a specificity of $90.6 \%$ in predicting the presence of initial distant metastases. ${ }^{16}$ This finding is supported by a larger retrospective study among 4029 DTC patients, which revealed a significant association between the magnitude of elevation of the pre-operative serum thyroglobulin level/concentration with the tumor burden and extent, i.e., the presence of lymph node involvement and distant metastases. ${ }^{17}$ Another study in Egypt revealed that a high pre-operative Tg level was helpful in the initial prognostication of DTC patients. ${ }^{18}$ Alternatively, a review of 598 DTC patients in the USA found that although the pre-operative value was associated with the size of the thyroid gland and tumor stages, it had a poor sensitivity $(10.3 \%)$ to detect distant metastasis. ${ }^{19}$

Following total thyroidectomy, the concentration of serum $\mathrm{Tg}$ should decline to a very low or undetectable level. As Tg is organ-specific, measuring its concentration after surgery is useful for the: 1) assessment of the completeness of surgical removal of the gland, 2) treatment guidance with radioactive iodine (RAI), and 3) disease prognostication. Surgical removal is considered successful if $\mathrm{Tg}$ remains undetectable, meanwhile a persistent high $\mathrm{Tg}$ level in circulation reflects the presence of residual disease or an incomplete surgical removal of the thyroid gland. A comparison study between thyroid scintigraphy and serum
Tg level in DTC patients showed that measurable $\mathrm{Tg}$ is more accurate in detecting residual tissue..$^{20}$

The American Thyroid Association guideline suggests the use of post-operative serum Tg level for treatment guidance as well as to inform the use of RAl therapy. ${ }^{2}$ A retrospective study in the USA among 134 patients undergoing total thyroidectomy, which compared patients who received RAI with those who did not, found no significant structural tumor recurrence in $49.3 \%$ of patients who had an initial undetectable post-operative serum $\mathrm{Tg}$. Patients with a serum $\mathrm{Tg}$ concentration between 0.2 and $2.0 \mathrm{ng} / \mathrm{ml}$ did not benefit from RAI therapy, while those with values beyond $2.0 \mathrm{ng} / \mathrm{ml}$ did not respond to RAl alone. ${ }^{21}$ Treatment is considered successful (excellent response) if Tg remains undetectable in the patient's circulation in the presence of negative imaging results. ${ }^{2}$ Consequently, the treatment intensity and frequency in such patients can be tapered down. Alternatively, rising post-operative serum Tg concentrations despite treatment and the presence of measurable $\mathrm{Tg}$ after previously undetected levels suggest the possibility of disease progression or recurrence, thus requiring further investigations and potential additional therapies.

In addition, the post-operative serum $\mathrm{Tg}$ level can be used as a prognostic marker. An excellent response to therapy is defined as persistent undetectable $\mathrm{Tg}$; this carries an excellent prognosis and is associated with a low recurrence rate and $a<1.0 \%$ disease-specific death rate. ${ }^{22}$ An observational study in Saudi Arabia reported that 98.0\% of DTC patients with a serum $\mathrm{Tg}$ concentration below 0.1 $\mathrm{ng} / \mathrm{ml}$ estimated at 3-6 months post-total thyroidectomy and RAl had no structural or functional disease, which indicates a very low risk of cancer recurrence. ${ }^{23}$ This finding was supported by another study that found a significant structural recurrence rate, which was high (30.0\%) among DTC patients with a stimulated post-operative thyroglobulin 
level of more than $2 \mathrm{ng} / \mathrm{ml}^{24}$ On the other hand, a retrospective study in 570 DTC patients demonstrated that a low or undetectable postoperative $\mathrm{Tg}$ level did not rule out metastases in early stage malignancy; $90.2 \%$ of metastatic patients had serum $\mathrm{Tg}$ levels below the detection limit. ${ }^{25}$

\section{Clinical aspects of anti-thyroglobulin antibody}

$\mathrm{TgAb}$ presents the most important interference that limits the clinical utility of $\mathrm{Tg}$ measurement. In circulation, $\operatorname{TgAb}$ binds to the circulating $\mathrm{Tg}$ and enhances the hepatic clearance compared to the free $\mathrm{Tg}$ alone. As a consequence, the $\mathrm{Tg}$ concentration is inappropriately low even in the presence of a high tumor burden. Likewise, it can also directly interfere with the immunoassay measurement, where it forms a complex with $\mathrm{Tg}$ in the patient's serum and prevents its binding to the capture and/ or signal antibody and, thus, generating a falsely low or high serum $\mathrm{Tg}$ value depending on the type of assay used. Any discordance in the results produced by different assay methods is suggestive of $\mathrm{TgAb}$ interference. While persistent undetectable $\mathrm{Tg}$ is classified as excellent response, the presence of $\mathrm{TgAb}$ nullifies it, and the patient will be regarded as having an intermediate level of response. ${ }^{2}$

Unlike $\mathrm{Tg}$, pre-operative $\mathrm{TgAb}$ has some clinical advantages. An elevated TgAb concentration in patients presenting with thyroid nodules is highly predictive of DTC. ${ }^{26}$ Thyroid cancer cells may produce a different Tg with novel immunogenic cancer epitopes that can induce $\mathrm{TgAb}$ synthesis. However, its elevated concentration is not specific to DTC, as it may also be found in thyroid autoimmune disease. Other than that, in DTC-diagnosed cases, the preoperative TgAb level helps to predict the prognosis of DTC. A positive $\operatorname{TgAb}$ result has been associated with a higher risk of persistent or recurrent DTC compared to patients with negative $\operatorname{TgAb}$ results. Several studies have also reported a correlation between a positive TgAb finding and a highly invasive tumor profile with lymph node metastases.
The post-operative serum TgAb test values can replace the $\mathrm{Tg}$ laboratory findings in the detection of disease persistence and recurrence. ${ }^{27}$ Typically, a transient increase in $\operatorname{TgAb}$ concentration is observed immediately after thyroidectomy in response to the spillage of a meager volume of thyroid tissue into circulation. Subsequently, its concentration is gradually reduced to naught. ${ }^{28}$ Thus, any appearance of $\operatorname{TgAb}$ or incremental increase in the $\operatorname{TgAb}$ titer postoperatively is suggestive of incomplete biochemical response. $^{2}$ On top of that, the TgAb level is useful as a surrogate marker for disease prognosis. A persistently high $\operatorname{TgAb}$ concentration post-therapy is associated with a poor treatment response and, therefore, an unfavorable prognosis. ${ }^{29,30}$ A retrospective study among 98 patients treated for DTC showed that stable as well as rising $\mathrm{TgAb}$ concentrations are associated with disease persistence or recurrence (Table 1$){ }^{31}$

\section{Laboratory aspects of thyroglobulin and}

\section{thyroglobulin antibody}

The serum $\mathrm{Tg}$ measurement has become fundamental to the post-operative monitoring of DTC patients. Thus, optimizing the relevant pre-analytical variables may improve the interpretation of serum $\mathrm{Tg}$. The half-life of serum $\mathrm{Tg}$ is approximately 1 to 3 days in post thyroidectomy ${ }^{32,33}$, reaching an undetectable concentration at 12 weeks. $^{34}$ Thus, the sample collection should be delayed until more than 4 to 6 weeks post-thyroid procedures to ensure an accurate interpretation of the disease status. It is also important to note whether the sample is taken under TSH stimulation or thyroid suppression therapy. TSH stimulation increases serum Tg, while suppression using levothyroxine lowers the serum $\mathrm{Tg}$ concentration. Therefore, concomitant measurement of serum TSH with serum Tg can assist in the interpretation of the $\mathrm{Tg}$ laboratory result. 
Table 1 The role of thyroglobulin and thyroglobulin antibody in differentiated thyroid carcinoma management

\begin{tabular}{|c|c|c|}
\hline & Thyroglobulin & Thyroglobulin antibody \\
\hline \multicolumn{3}{|l|}{ Preoperative concentration } \\
\hline 1. Risk of malignancy & $\begin{array}{l}\text { Values above certain cut-off levels predict risk } \\
\text { of thyroid malignancy, but require further } \\
\text { validation study }\end{array}$ & $\begin{array}{l}\text { High predictive value for DTC in thyroid } \\
\text { nodules, but not specific }\end{array}$ \\
\hline 2. Tumor staging & $\begin{array}{l}\text { Degree of elevation is associated with tumor } \\
\text { size, lymph node involvement, and presence } \\
\text { of metastases }\end{array}$ & $\begin{array}{l}\text { Associated with higher risk of persistent or } \\
\text { recurrent DTC, invasive tumor profile, and } \\
\text { lymph node metastases }\end{array}$ \\
\hline \multicolumn{3}{|l|}{ Postoperative concentration } \\
\hline $\begin{array}{l}\text { 1. Assessment of completeness of } \\
\text { surgical removal }\end{array}$ & $\begin{array}{l}\text { Level reflects presence of residual disease or } \\
\text { incomplete surgical removal }\end{array}$ & $\begin{array}{l}\text { Appearance or incremental presence is } \\
\text { suggestive of incomplete biochemical } \\
\text { response }\end{array}$ \\
\hline 2. Treatment guidance & $\begin{array}{l}\text { - Below lower limit of detection: may or may } \\
\text { not receive RAl. } \\
\text { - } 0.2 \text { to } 2.0 \mathrm{ng} / \mathrm{ml} \text { : benefit from RAl therapy } \\
\text { modalities } \\
\text { - Downward trend to persistent undetect } \\
\text { able concentration: excellent response, } \\
\text { and treatment intensity and frequency can } \\
\text { be tapered down }\end{array}$ & None \\
\hline 3. Prognostication & $\begin{array}{l}\text { Persistent undetectable level is associated } \\
\text { with low risk of tumor recurrence }\end{array}$ & $\begin{array}{l}\text { Persistently high level post-treatment is } \\
\text { associated with poor treatment response } \\
\text { and an unfavourable prognosis }\end{array}$ \\
\hline
\end{tabular}

There are various ways to measure $\mathrm{Tg}$ level; it was first measured via competitive immunoassay, e.g., radioimmunoassay $(R \mid A)$. However, this method is rarely adopted in clinical practice due to difficulties related to the proper disposal of radioactive waste as well as the short shelf-life and instability of the radio-labelled reagent. ${ }^{35}$ Currently, the immunometric $\mathrm{Tg}$ assays (IMA) are widely used in clinical laboratories because they are amenable to automation allowing for the generation of many results with a short turnaround time. ${ }^{36}$ Nevertheless, they are susceptible to 1) interferences from TgAb or heterophile antibodies, 2) hook effects, and 3) poor inter-platform concordance.

Recently, mass spectrometric (MS) methods have emerged as a promising modalities to overcome the immunoassay analytical challenges, particularly $\mathrm{TgAb}$ interference, in DTC patients. ${ }^{37}$ They eliminate the use of antibodies for $\mathrm{Tg}$ detection; instead, they measure $\mathrm{Tg}$ according to its molecular-weight-to-ionic-charge ratio.
The combination of MS with high performance liquid chromatography increases its specificity. However, despite its good specificity, this method has a lower sensitivity compared to immunoassays, which renders it unsuitable for the monitoring of DTC patients. ${ }^{38,39}$ Furthermore, it is technically demanding, has a long turnaround time, and requires trained laboratory technologists.

Given the variety of methods available, it is required for the manufacturers to certify that the functional sensitivity (FS) of their assay is $<1 \mathrm{ng} / \mathrm{ml}$. FS is defined as the lowest concentration of $\mathrm{Tg}$ that can be measured with a coefficient variation of $20 \%{ }^{40}$ This is especially important in low-risk patients on levothyroxine because the result reflects the presence of residual disease. In addition, a highly sensitive assay is able to detect the presence of small amounts of thyroid tissue without the need for $\mathrm{TSH}$ stimulation. At present, newer $\mathrm{Tg}$ assays with an improved sensitivity $(\mathrm{FS}<$ $0.1 \mathrm{ng} / \mathrm{mL}$ ) are becoming more available. This improvement 
may raise the positive predictive value at the cost of reducing the negative predictive value. Therefore, serial measurements of $\mathrm{Tg}$ reflect more on the disease status compared to a single result.

The vast diversity of $\mathrm{Tg}$ epitopes complicates the development of highly specific $\mathrm{Tg}$ antibodies and, thus, results in different $\mathrm{Tg}$ isoform measurements. ${ }^{41}$ Different assays utilize different antibodies, which target different epitopes to quantify $\mathrm{Tg}$, resulting in concentration discrepancies between different analytical platforms. For example, the use of polyclonal antibodies in RIAs allows the system to measure a wider range of $\mathrm{Tg}$ isoforms. Contrastingly, the measurement of $\mathrm{Tg}$ via monoclonal antibodies in IMA produces a lower result due to the antibodies' limited specificity for the Tg epitopes. Despite the international efforts to standardize all the analytical methods by calibrating them against the Certified Reference Materials (BCR 457, European Commission, Institute of Reference Materials) ${ }^{42,43}$, a significant inter-assay bias is still present. ${ }^{37}$ Thus, a few international guidelines recommend the use of the same $\mathrm{Tg}$ assay in a given laboratory for the monitoring of patients with DTC. ${ }^{2,44,45}$

Current guidelines mandate the measurement of $\mathrm{TgAb}$ with every $\mathrm{Tg}$ testing in order to identify potential interference. Previously, the methods for quantifying $\operatorname{TgAb}$ have consisted of 1) semi-quantitative assays, which measure the precipitation of $\mathrm{TgAb}$ in serum with spiked-labelled $\mathrm{Tg}$, and 2) indirect assays, e.g. the recovery test, which measures the expected recovery of the exogenous $\mathrm{Tg}$ that is added to the patient's serum. However, the low sensitivity of these methods limits their usage in clinical practice. Currently, the gold standard method for the measurement of TgAb is IMA assays, which are standardized in accordance with the International Reference Preparation and Medical Research Council 65/93 guidelines. Nevertheless, despite the standardization efforts, a significant inter-assay discordance persists due to the variable degree of $\mathrm{TgAb}$ epitopic specificities to $\mathrm{Tg}$. At present, there is no established value at which $\operatorname{TgAb}$ interferes with the $\mathrm{Tg}$ measurement. High concentrations of $\operatorname{TgAb}$ do not always associate with assay interference, and conversely, low TgAb levels may result in strong assay interference. $^{37}$

Linearity is another issue in the $\mathrm{Tg}$ measurement, especially in metastatic DTC with extremely high $\mathrm{Tg}$ concentrations. When the concentration of $\mathrm{Tg}$ in the serum is too high, it can saturate both the capture and signal antibodies and, hence, prevent the sandwich formation in IMA. This high-dose hook effect is common in a homogenous assay, in which there is no additional washing step; this results in an erroneously low Tg level in patients with a very high analyte concentration. ${ }^{46}$ Consequently, that leads to the misinterpretation of the result. The simultaneous measurement of the original and diluted patient samples may reduce the occurrence of this phenomenon; although, this may impose an additional cost to the laboratory. A recent case in Amsterdam reported a discordance of the $\mathrm{Tg}$ level with the clinical condition due to the presence of the high-dose hook effect; this proves the persistence of this phenomenon in current practice despite the approaches undertaken to minimize it. ${ }^{46}$

The current available reference interval for the Tg level interpretation is derived from normal population individuals with healthy thyroid glands and a TSH concentration within the normal reference range. However, because the aim of $\mathrm{Tg}$ measurement in DTC monitoring is to observe the trend of serum Tg level and to keep its concentration below the detection limit, comparing its value to the normal range becomes less important.

Apart from the measurement of $\mathrm{Tg}$ in the serum, it's utility as a immunohistochemical marker for thyroid carcinoma, particularly in DTCs, is well established. ${ }^{47}$ Ninety-eight percent of papillary thyroid carcinoma (PTC) cases and $95.2 \%$ of patients with follicular thyroid carcinoma 
(FTC) demonstrate positive $\mathrm{Tg}$ staining. ${ }^{48}$ Generally, both PTC and FTC show strong and diffuse cytoplasmic staining with intense Tg expression at the luminal colloid. ${ }^{49}$ However, the staining intensity and pattern are heterogenous between PTC and FTC as well as within the same tumor subtype. FTC exhibits more immunoreactivity compared to PTC, and it often shows a more uniform staining pattern. ${ }^{50}$ The staining intensity in both types of DTC reveals a significant correlation with the degree of tumor differentiation-well-differentiated tumors display strong Tg staining, while poorly-differentiated tumors may lose their immunoreactivity. ${ }^{50} \mathrm{Tg}$ expression can also be altered in different tumor subtypes. For example, the columnar cell variant of PTC, and the hobnail cell variant of PTC are Tg-immunoreactive, but the cribriform morular variant of PTC is often Tg-negative. Similar findings can also be seen in clear cell FTC. ${ }^{51}$ Another factor influencing Tg expression in DTC is iodine avidity; a 2021 study in Sweden among 45 confirmed PTC patients reported that the percentage of cells expressing $\mathrm{Tg}$ is strongly correlated to iodine avidity. ${ }^{52}$

\section{Conclusion}

The clinical significance of $\operatorname{Tg}$ and $\operatorname{TgAb}$ concentration is confirmed by the findings of this study. The accurate measurement of serum $\mathrm{Tg}$ and reliable detection of $\operatorname{TgAb}$ are pertinent to the follow-up of DTC patients. The use of a gold standard method should be adopted by laboratories, and suspicious results should be interpreted and managed in parallel with clinical findings.

\section{Conflict of interest}

There are no potential conflicts of interest to declare.

\section{Reference}

1. Bray F, Ferlay J, Soerjomataram I, Siegel RL, Torre LA, Jemal A. Global cancer statistics 2018: GLOBOCAN estimates of incidence and mortality worldwide for 36 cancers in 185 countries. CA Cancer Clin 2018;68:394-424.
2. Haugen BR, Alexander EK, Bible KC, Doherty GM, Mandel SJ, Nikiforov YE, et al. 2015 American Thyroid Association management guidelines for adult patients with thyroid nodules and differentiated thyroid cancer: the American Thyroid Association guidelines task force on thyroid nodules and differentiated thyroid cancer. Thyroid 2016;26:1-133.

3. Mitchell A, Gandhi A, Scott-Coombes D, Perros P. Management of thyroid cancer: United Kingdom national multidisciplinary guidelines. J Laryngol Otol 2016;130:S150-60.

4. Evans $C$, Tennant S, Perros P. Thyroglobulin in differentiated thyroid cancer. Clinica Chimica Acta 2015;444:310-7.

5. Bilek R, Dvorakova M, Grimmichova T, Jiskra J. lodine, thyroglobulin and thyroid gland. Physiol Res 2020;69(Suppl 2): s225-36.

6. Xavier ACW, Maciel R, Vieira JGH, Dias-da-Silva MR, Martins JR. Insights into the posttranslational structural heterogeneity of thyroglobulin and its role in the development, diagnosis, and management of benign and malignant thyroid diseases. Arch Endocrinol Metab 2016;60:66-75.

7. Citterio CE, Targovnik HM, Arvan P. The role of thyroglobulin in thyroid hormonogenesis. Nat Rev Endocrinol 2019;15:32338.

8. Clark P, Franklyn J. Can we interpret serum thyroglobulin results? Ann Clin Biochem 2012;49:313-22.

9. Doggui R, editor. Immunoanalytical profile of thyroglobulin antibodies. Ann Bio Clin (Paris) 2018;76:695-704.

10. Chiovato L, Latrofa F, Braverman LE, Pacini F, Capezzone M, Masserini L, et al. Disappearance of humoral thyroid autoimmunity after complete removal of thyroid antigens. Ann Intern Med 2003;139:346-51.

11. D’Aurizio F, Metus P, Ferrari A, Caruso B, Castello R, Villalta $D$, et al. Definition of the upper reference limit for thyroglobulin antibodies according to the National Academy of Clinical Biochemistry guidelines: comparison of eleven different automated methods. Auto Immun Highlights 2017;8: $1-10$.

12. Hulikal N, Azhagamuthu R, Banoth M, Chowhan AK, Yutla M, Sachan A. Can preoperative serum thyroglobulin levels predict the risk of malignancy? Results from prospective analysis of biochemical predictors of malignancy in thyroid nodules. Acta Otorhinolaryngol Ital 2020;40:33.

13. Kars A, Aktan B, Kilic K, Sakat MS, Gözeler MS, Yörük Ö, 
et al. Preoperative serum thyroglobulin level as a useful predictive marker to differentiate thyroid cancer. ORL 2018;80: 290-5.

14. Wang Z, Kang J, Guo B, Deng X, Bo W, Fan Y. Preoperative serum thyroglobulin in prediction of follicular thyroid tumors. Chin J Endocr Surg 2017;11:391-4.

15. Kim HJ. Updated guidelines on the preoperative staging of thyroid cancer. Ultrasonography 2017;36:292.

16. Kim H, Kim YN, Kim HI, Park SY, Choe JH, Kim JH, et al. Preoperative serum thyroglobulin predicts initial distant metastasis in patients with differentiated thyroid cancer. Sci Rep 2017;7:1-7.

17. Kim H, Park SY, Choe JH, Kim JS, Hahn SY, Kim SW, et al. Preoperative serum thyroglobulin and its correlation with the burden and extent of differentiated thyroid cancer. Cancers 2020;12:625.

18. Rageh TM, Abdou AG, Elkhouly EA, Abou El-Ela DH, Zidan MA. Preoperative significance of thyroglobulin, thyroid stimulating hormone and thyroglobulin antibody in differentiated papillary thyroid carcinoma. Int Surg J 2019;6:4229-37.

19. Patell R, Mikhael A, Tabet M, Bena J, Berber E, Nasr C. Assessing the utility of preoperative serum thyroglobulin in differentiated thyroid cancer: a retrospective cohort study. Endocrine 2018; 61:506-10.

20. Atilgan $\mathrm{HI}$, Yalcin $\mathrm{H}$. Estimation of residual tissue with thyroid scintigraphy and thyroglobulin level in the pre-ablative period differentiated thyroid carcinoma. Ulutas Med J 2020; 6:169-75.

21. McDow AD, Shumway CM, Pitt SC, Schneider DF, Sippel RS, Long KL. Utility of early postoperative unstimulated thyroglobulin in influencing decision making in patients with papillary thyroid carcinoma. Ann Surg Oncol 2019;26:4002-7.

22. Prpić M, Franceschi M, Romić M, Jukić T, Kusić Z. Thyroglobulin as a tumor marker in differentiated thyroid cancerclinical considerations. Acta Clinica Croatica 2018;57:518-26.

23. Jammah AA, Masood A, Akkielah LA, Alhaddad S, Alhaddad MA, Alharbi M, et al. Utility of stimulated thyroglobulin in reclassifying low risk thyroid cancer patients' following thyroidectomy and radioactive iodine ablation: a 7 -year prospective trial. Front Endocrinol 2020;11.

24. Jayasekara J, Jonker P, Lin JF, Engelsman AF, Wong MS, Kruijff $S$, et al. Early postoperative stimulated serum thyro- globulin quantifies risk of recurrence in papillary thyroid cancer. Surgery 2020;167:40-5.

25. Campennì A, Giovanella L, Pignata SA, Vento A, Alibrandi A, Sturiale $L$, et al. Undetectable or low $(<1 \mathrm{ng} / \mathrm{ml})$ postsurgical thyroglobulin values do not rule out metastases in early stage differentiated thyroid cancer patients. Oncotarget 2018; 9:17491.

26. Alhefdhi A, Altayyar T, Alqahtani SM, Alshehri M, Elnaas S, Alsobhi $\mathrm{S}$. Can preoperative serum thyroglobulin and antithyroglobulin levels predict malignant potential of a thyroid nodule with atypia or follicular lesion of undetermined significance?. Otorhinolaryngol Head Neck Surg 2020:5:1-4

27. Drake T. Serum antithyroglobulin antibodies predict differentiated thyroid cancer persistence/recurrence and Mortality. Clinical Thyroidology 2020;32:344-6.

28. Matrone A, Latrofa F, Torregrossa L, Piaggi P, Gambale C, Faranda $A$, et al. Changing trend of thyroglobulin antibodies in patients with differentiated thyroid cancer treated with total thyroidectomy without 1311 ablation. Thyroid 2018;28:871-9.

29. Ernaga-Lorea A, Hernandez-Morhain M, Anda-Apinaniz E, Pineda-Arribas J, Migueliz-Bermejo I, Eguilaz-Esparza N, et al. Prognostic value of change in anti-thyroglobulin antibodies after thyroidectomy in patients with papillary thyroid carcinoma. Clin Transl Oncol 2018;20:740-4.

30. Lee ZJO, Eslick GD, Edirimanne S. Investigating antithyroglobulin antibody as a prognostic marker for differentiated thyroid cancer: a meta-analysis and systematic review. Thyroid 2020;30:1601-12.

31. Reverter JL, Rosas-Allende I, Puig-Jove C, Zafon C, Megia A, Castells I, et al. Prognostic significance of thyroglobulin antibodies in differentiated thyroid cancer. J Thyroid Res 2020; 2020.

32. Hocevar M, Auersperg M, Stanovnik L. The dynamics of serum thyroglobulin elimination from the body after thyroid surgery. Eur J Surg Oncol 1997;23:208-10.

33. Giovanella L, Ceriani L, Maffioli M. Postsurgery serum thyroglobulin disappearance kinetic in patients with differentiated thyroid carcinoma. Head Neck 2010;32:568-71.

34. Patel A, Shostrom V, Treude K, Lydiatt W, Smith R, Goldner W. Serum thyroglobulin: preoperative levels and factors affecting postoperative optimal timing following total thyroidectomy. Int J Endocrinol 2019;2019. 
35. Spencer CA, LoPresti JS. Technology Insight: measuring thyroglobulin and thyroglobulin autoantibody in patients with differentiated thyroid cancer. Nat Clin Pract Endocrinol Metab 2008;4:223-33.

36. Algeciras-Schimnich A. Thyroglobulin measurement in the management of patients with differentiated thyroid cancer. Crit Rev Clin Lab Sci 2018;55:205-18.

37. Netzel BC, Grebe SK, Carranza Leon BG, Castro MR, Clark PM, Hoofnagle AN, et al. Thyroglobulin ( $\mathrm{Tg}$ ) testing revisited: $\mathrm{Tg}$ assays, $\operatorname{TgAb}$ assays, and correlation of results with clinical outcomes. J Clin Endocrinol Metab 2015;100:E1074-E83.

38. Azmat U, Porter K, Senter L, Ringel MD, Nabhan F. Thyroglobulin liquid chromatography-tandem mass spectrometry has a low sensitivity for detecting structural disease in patients with antithyroglobulin antibodies. Thyroid 2017;27:74-80.

39. Shuford CM, Johnson JS, Thompson JW, Holland PL, Hoofnagle AN, Grant RP. More sensitivity is always better: measuring sub-clinical levels of serum thyroglobulin on a $\mu \mathrm{LC}-\mathrm{MS} / \mathrm{MS}$ system. Clinical Mass Spectrometry 2020;15:29-35.

40. Baloch Z, Carayon P, Conte-Devolx B, Demers LM, FeldtRasmussen U, Henry JF, et al. Laboratory medicine practice guidelines. Laboratory support for the diagnosis and monitoring of thyroid disease. Thyroid 2003;13:3-126.

41. Giovanella L, Feldt-Rasmussen U, Verburg FA, Grebe SK, Plebani M, Clark PM. Thyroglobulin measurement by highly sensitive assays: focus on laboratory challenges. Clin Chem Lab Med 2015;53:1301-14.

42. Feldt-Rasmussen U, Profilis C, Colinet E, Black E, Bornet $H$, Bourdoux $\mathrm{P}$, et al, editors. Human thyroglobulin reference material (CRM 457). 1st part: assessment of homogeneity, stability and immunoreactivity. Ann Bio Clin (Paris) 1996;54: 337-42.

43. Feldt-Rasmussen $U$, Profilis $\mathrm{C}$, Colinet $\mathrm{E}$, Black E, Bornet $\mathrm{H}$, Bourdoux $\mathrm{P}$, et al, editors. Human thyroglobulin reference material
(CRM 457). 2nd part: physicochemical characterization and certification. Ann Bio Clin (Paris) 1996;54:343-8.

44. Filetti S, Durante C, Hartl D, Leboulleux S, Locati L, Newbold K, et al. Thyroid cancer: ESMO Clinical Practice Guidelines for diagnosis, treatment and follow-up. Ann Oncol 2019;30: 1856-83.

45. Fugazzola L, Elisei R, Fuhrer D, Jarzab B, Leboulleux S, Newbold K, et al. 2019 European Thyroid Association guidelines for the treatment and follow-up of advanced radioiodinerefractory thyroid cancer. Euro Thyroid J 2019;8:227-45.

46. Hillebrand J, Siegelaar S, Heijboer A. Falsely decreased thyroglobulin levels in a patient with differentiated thyroid carcinoma. Clinica Chimica Acta 2020;509:217-9.

47. Whitley RJ, Ain KB. Thyroglobulin: a specific serum marker for the management of thyroid carcinoma. Clin Lab Med 2004;24:29-47.

48. Steurer S, Schneider J, Büscheck F, Luebke AM, Kluth M, Hube-Magg C, et al. Immunohistochemically detectable thyroglobulin expression in extrathyroidal cancer is $100 \%$ specific for thyroidal tumor origin. Ann Diagn Pathol 2021;54:151793.

49. Baloch Z, Mete O, Asa SL. Immunohistochemical biomarkers in thyroid pathology. Endocr Pathol 2018;29:91-112.

50. Ryff-de Lèche A, Staub JJ, Kohler-Faden R, Müller-Brand JA, Heitz PU. Thyroglobulin production by malignant thyroid tumors. An immunocytochemical and radioimmunoassay study. Cancer 1986;57:1145-53.

51. Cameselle-Teijeiro JM, Eloy C, Sobrinho-Simões M. Pitfalls in challenging thyroid tumors: emphasis on differential diagnosis and ancillary biomarkers. Endocr Pathol 2020;31:197-217.

52. Nilsson JN, Siikanen J, Hedman C, Juhlin CC, Ihre Lundgren C. Pre-therapeutic measurements of lodine avidity in papillary and poorly differentiated thyroid cancer reveal associations with thyroglobulin expression, histological variants and $\mathrm{Ki}-67$ index. Cancers 2021;13:3627. 